\title{
Structural and magnetic investigations of new skyrmion phases
}

Geetha Balakrishnan ${ }^{1}$, Monica Ciomaga Hatnean ${ }^{1}$, Ales Stefancic ${ }^{1}$, Martin R Lees ${ }^{1}$

${ }^{1}$ Department Of Physics, University Of Warwick, Coventry, United Kingdom E-mail: g.balakrishnan@warwick.ac.uk

Recent studies report the existence of skyrmions in materials with a different chiral space group from that of the B20 compounds, such as GaV4S8 [1] and Co10-xZn10-yMnx+y [2]. This has motivated us to embark upon a study of several classes of skyrmion materials and explore the existence of the skyrmion phase in a wide composition range of each of the above family of compounds: i.e., GaV4X8 (X=S, Se, Te), Co10-xZn10-yMnx+y and the Cu2-xAxOSeO3 ( $A=\mathrm{Zn}, \mathrm{Ni})$.

GaV4S8 crystallizes in a lacunar spinel non-centrosymmetric cubic F-43m structure at room temperature and orders ferromagnetically below $\mathrm{Tc}=13 \mathrm{~K}$ below which it has a rather puzzling magnetic phase diagram. A skyrmion state has been suggested to appear when a very small magnetic field (10-100 mT) is applied in the temperature range 8 to $13 \mathrm{~K}$ [1]. Co10$x Z n 10-y M n x+y$ alloys belong to a family of cubic chiral ferromagnets (Tc between 150 to $420 \mathrm{~K}$ ) that crystallise in the B-Mntype structure. A recent study reports on the formation of a skyrmion phase in this system at and above room temperature, under application of a very small magnetic field of $10 \mathrm{mT}$ [2]. In the well studied ferrimagnet $\mathrm{Cu} 20 \mathrm{SeO} 3$ (TN=56 $\mathrm{K}$ ), substitutions at the two different $\mathrm{Cu}(\mathrm{I})$ and $\mathrm{Cu}(\mathrm{II})$ sites by both magnetic and non-magnetic atoms ( $\mathrm{Zn}, \mathrm{Ni})$ [3] sheds light on the origin, the formation, and tuning of the skyrmion lattices.

A number of the above materials have been synthesized and structural investigations have been carried out using both powder and single crystal X-ray diffraction. A study of the magnetic properties of these materials has also been carried out by ac and dc magnetic susceptibility measurements. We present a detailed structural and magnetic study of these interesting classes of materials demonstrating the structural similarities of these materials and the correlation with their magnetic properties.

[1] Kézsmárki, I. (2015). Nat. Mater. 14, 1116.

[2] Tokunaga, Y. (2015). Nat. Commun. 6, 7638.

[3] Wu, H. C. (2015). Sci. Rep. 5, 13579.

Keywords: Skyrmions, Skyrmion Phases, Magnetic skyrmions 